\title{
IL SECONDO LIBRO DE MOTTETTI A UNA E DUE VOCI DI ANTONIO GUALTIERI E IL PARNASSUS MUSICUS FERDINANDAEUS
}

\author{
CHIARA COMPARIN \\ Università degli Studi di Padova
}

\begin{abstract}
Izvleček: Razprava osvetljuje glavne značilnosti zbirke Antonia Gualtierija iz leta 1612 in vzporeja nekatere motete iz te zbirke s tistimi Gualtierijevimi moteti, objavljenimi v antologiji Parnassus musicus Ferdinandaeus, ki imajo enako zasedbo in kažejo podobne kompozicijske značilnosti. Ključne besede: Antonio Gualtieri, mali duhovni koncerti, 17. stoletje.
\end{abstract}

\begin{abstract}
This paper illustrates the main features of Antonio Gualtieri's collection of 1612 and compares some of Gualtieri's motets with certain compositions included in the Parnassus Musicus Ferdinandaeus that feature the same scoring and exhibit similar compositional procedures. Keywords: Antonio Gualtieri, small-scale motets, seventeenth century.
\end{abstract}

Antonio Gualtieri rientra in una categoria di musicisti sinora relegati ai margini della ricerca a dispetto dell'interesse musicale della loro produzione. ${ }^{1}$ Nel presente contributo intendo mettere in luce alcuni risultati emersi in tempi più recenti, ${ }^{2}$ concentrandomi in particolare sul suo secondo libro di mottetti a una e due voci stampato a Venezia nel 1612, soltanto tre anni prima della raccolta Parnassus musicus Ferdinandaeus, e proponendo alcuni confronti fra le composizioni di Gualtieri e quelle incluse nel Parnassus che presentano lo stesso organico. Ho scelto di limitare il confronto ai mottetti a voce sola e basso continuo e ai mottetti a due voci che presentano lo stesso organico in considerazione del fatto che, dei nove mottetti a due voci e basso continuo della raccolta di Gualtieri, ben sette presentano due voci pari, mentre è del tutto assente la combinazione soprano e basso, la quale invece risulta prevalente all'interno del Parnassus. ${ }^{3}$

Nato a Monselice nel 1574, Antonio Gualtieri compì i suoi studi presso la cattedrale di Padova e, dopo un periodo di attività come organista e maestro di cappella presso

1 Dizionario Enciclopedico della Musica e dei Musicisti, s.v. "Gualtieri, Antonio"; J. Roche e E. Roche, "Gualtieri, Antonio", 472-473; Morche, "Gualtieri, Antonio", 140-142; Colussi, "Gualtieri, Antonio", 1385-1388; Colussi, "Tracce di musica policorale", 101-158; Comparin, "Antonio Gualtieri"; Lovato, "Musica e liturgia", 231-249; Passadore, Musica e musicisti a Rovigo, 48-56.

2 Comparin, "Antonio Gualtieri".

3 Nel presente contributo sono quindi 14 i mottetti tratti dal Parnassus musicus Ferdinandaeus oggetto delle nostre considerazioni: Antonicek, ed., Parnassus musicus Ferdinandaeus, 1-22, 84-104, 107-110. 
diversi centri della Serenissima (San Daniele del Friuli, Monselice, Montagnana), nel 1632 approdò a Venezia, dove venne assunto dapprima presso l'Ospedale della Pietà con il compito di "insegnar di musica et sonar alle fie di questo loco" e, qualche anno più tardi, venne eletto "maestro delli zaghi della chiesa di San Marco" e maestro dei chierici del seminario di San Marco. Gualtieri si trovò dunque a operare in una delle più prestigiose istituzioni musicali del XVII secolo negli stessi anni in cui Claudio Monteverdi ne dirigeva la cappella musicale. ${ }^{4}$

La produzione musicale di Gualtieri, a quanto ci consta, doveva comprendere complessivamente sette raccolte, tutte stampate a Venezia tra il 1604 e il 1630: i Motecta octonis vocibus (1604), gli Amorosi diletti a tre voci (1608), i Mottecta duabus vocibus (1611) menzionati da Robert Eitner e attualmente non reperibili, ${ }^{5}$ Il secondo libro de mottetti a una e due voci (1612), Il secondo libro de' madrigali a cinque voci (1613), i Madrigali concertati a una, due et tre voci (1625) e i Mottetti a una, doi, tre et quatro voci con le littanie della B. Vergine a 4 (1630). ${ }^{6}$

Anche a un primo sguardo, possiamo osservare che l'opera di Gualtieri si inserisce nel clima culturale del primo Seicento, dal momento che il compositore si è dedicato ai generi allora più diffusi: nella produzione profana si cimenta con i generi di maggior fortuna, come la canzonetta e il madrigale concertato; in quella sacra, invece, facendo proprie le diverse esperienze degli anni di formazione presso la cattedrale di Padova e di attività come maestro di cappella, egli spazia dai mottetti a otto voci, legati alla produzione policorale diffusa in area veneta - e in particolare nell'area padovana - ai concerti a una, due, tre voci e basso continuo del 1612 e del 1630. In queste due raccolte Gualtieri approda allo stile concertato, arricchito dall'apporto strumentale di violini e tromboni e nella cui scrittura fa propri stilemi che ebbero probabilmente fra i loro modelli di riferimento i Cento concerti ecclesiastici (1601) di Lodovico Grossi da Viadana. Sappiamo infatti che Gualtieri, strettamente legato al vescovo Marco Cornaro, poteva essere entrato in contatto con le numerose musiche che questi era solito riportare a Padova dai suoi numerosi viaggi a Roma. ${ }^{7}$ A questo proposito non possiamo trascurare la grande importanza che Marco II Cornaro, vescovo di Padova dal 1595 al 1625, ebbe nella formazione musicale di Gualtieri e, più in generale, nella vita musicale padovana. Le diverse raccolte a lui dedicate, tra le quali si annovera anche il Completorium Romanum (Venezia, 1597) di Ludovico Grossi da Viadana, testimoniano l'importanza della sua presenza nel mondo musicale - e non solo in quello padovano - sia per le attività promosse, sia per la costante attenzione rivolta a cantori e musicisti. ${ }^{8}$ Gli interessi musicali del vescovo emergono chiaramente

4 Venezia, Archivio di Stato, Ospedali e luoghi pii diversi, bs. 892, Ospedale della Pietà, Terminazioni (1618-1655), c. 75rv; Procuratia de Supra Chiesa S. Marco, bs. 89, proc. 200, Maestro di canto (1485-1734), c. 10r e v; Procuratia de Supra del Seminario, bs. 156, proc. 316, Maestro di canto del Seminario di Castello (1579-1781), c. $7 v$.

5 Una copia era presente nella Biblioteca Universitaria di Königsberg, è oggi dispersa. Eitner, Quellen-Lexicon, 398-399; Müller, Die musikalischen Schätze, 189-190.

6 RISM G 4791-4796.

7 Lovato, "La musica sacra", 37-41.

8 La prima opera dedicata a Marco II Cornaro fu Il quinto libro di madrigali a 5 voci (Venezia, 1587) di Marc'Antonio Pordenon. Dieci anni più tardi, oltre alla già citata raccolta di Viadana, 
dalla dedicatoria dei Motecta octonis vocibus di Gualtieri, che, al di là delle consuete formule di circostanza che i musicisti rivolgevano a mecenati e protettori, esprime la sua riconoscenza per essere stato sostenuto nello studio. ${ }^{9}$

Le principali istituzioni musicali padovane dell'epoca erano la cappella della basilica di Sant'Antonio e la cappella della cattedrale. Come era consuetudine nelle più importanti realtà musicali del periodo, anche a Padova l'attività delle cappelle era arricchita dalla presenza di organisti, cantori e strumentisti. ${ }^{10}$ I documenti che riportano gli organici delle cappelle padovane attestano che, in occasione di particolari festività liturgiche, la formazione veniva ampliata da musicisti provenienti da altre città e nazioni. Allo stesso modo, nomi di musicisti padovani figuravano nelle cappelle musicali di altre importanti città. Nei primi quindici anni del Seicento, alla guida delle due cappelle padovane troviamo personalità come Costanzo Porta e Luigi Balbi (tab. 1). ${ }^{11}$

\section{Tabella 1}

Maestri di cappella della Basilica del Santo e della cattedrale di Padova nei primi trent'anni del Seicento

\begin{tabular}{|l|l|l|l|}
\hline \multicolumn{2}{|l|}{ Basilica del Santo } & \multicolumn{2}{l|}{ Cattedrale } \\
\cline { 1 - 2 } $1595-1601$ & Costanzo Porta & \multirow{2}{*}{$1598-1604$} & Lelio Bertani \\
\cline { 1 - 2 } $1601-1606$ & Bartolomeo Ratti & \\
\cline { 1 - 2 } $1606-1608$ & Giulio Belli & \multirow{2}{*}{ Bartolomeo Favaretto } \\
\cline { 1 - 2 } $1608-1613$ & Bartolomeo Ratti & \\
\cline { 1 - 2 } $1614-1615$ & Gian Antonio Filippini “da Cento" & & \multirow{2}{*}{ Girolamo Boni } \\
\cline { 1 - 2 } $1615-1621$ & Luigi Balbi & Antonio Vicentino \\
\hline $1621-1623$ & Giovanni Ghizzolo & & \\
\hline $1623-1631$ & Leandro Gallerano & & \\
\hline
\end{tabular}

Nelle loro raccolte musicali i maestri attivi a Padova in questo periodo prediligono il repertorio sacro, senza però trascurare quello profano, con raccolte di madrigali e canzonette (Appendice 1).

La frequente presenza di composizioni di musicisti attivi a Padova all'interno di numerose raccolte stampate in Italia e all'estero (Appendice 2) mostra come Padova non fosse un centro isolato, ma, al contrario, avesse una vivace vita musicale, come dimostra anche la cospicua circolazione di cantori e musicisti provenienti da altre realtà. Fra questi va ricordato Bartolomeo Barbarino, suonatore di chitarrone presso la basilica del Santo, musico del vescovo Marco Cornaro tra il 1606 e il 1608 e successivamente maestro di

vennero indirizzate al vescovo patavino anche la Vespertina omnium solemnitatum psalmodia di Giovanni Croce e l'Antiphonarium vespertinum di Girolamo Lambardi. A seguire i Motecta octonis vocibus (Venezia, 1604), che rappresenta la prima raccolta di Gualtieri, il Primo libro dei madrigali a 5 voci di Stefano Landi (Venezia, 1619) e le Messe parte per capella et parte per concerto di Giovanni Ghizzolo (Venezia, 1625).

9 Sull'importanza del vescovo Marco II Cornaro nella vita musicale di Padova: Garbelotto, Un vescovo musicista; Lovato, "La musica sacra".

${ }^{10}$ Cattin e Massaro, "La musica e le istituzioni musicali"; Morelli, "Il Seicento".

${ }^{11}$ Per il presente contributo si è scelto di porre come limite temporale il 1615, anno di pubblicazione del Parnassus. 
cappella presso la cattedrale, che troviamo rappresentato in diverse raccolte dell'epoca e anche all'interno del Parnassus musicus Ferdinandaeus con il suo mottetto Omnes sitientes. ${ }^{12}$ Un altro musicista legato all'ambiente padovano che trova spazio all'interno del Parnassus con due mottetti, Super flumina Babylonis (a tre voci, Canto, Tenore e Basso) e Confitemini gentes (a quattro voci), è il bresciano Giovanni Ghizzolo. Allievo di Costanzo Porta, Ghizzolo subentrò a Luigi Balbi alla guida della cappella musicale della basilica del Santo nel 1621 e vi rimase fino al 1623, quando si traferì a Novara e fu sostituito da Leandro Gallerano, suo concittadino. Alcuni mottetti dei due maestri bresciani verranno inclusi anche nella Ghirlanda sacra curata da Leonardo Simonetti e stampata a Venezia presso Bartolomeo Magni nel 1625. ${ }^{13}$ Nella stessa raccolta, oltre a un mottetto di Giovanni Rovetta e a quattro composizioni di Claudio Monteverdi, troviamo anche due mottetti di un altro allievo di Costanzo Porta, Amadio Freddi, cantore presso la cappella antoniana dal 1594 e successivamente maestro di cappella della cattedrale di Padova dal 1634 fino alla morte, avvenuta pochi mesi dopo la nomina.

In questo clima di vivace produzione vedono la luce le due raccolte di mottetti a poche voci e basso continuo di Antonio Gualtieri, edite rispettivamente nel 1612 e nel 1630. In questo periodo il compositore era attivo come maestro di cappella a Monselice e a Montagnana, due centri periferici nelle vicinanze di Padova che disponevano di cappelle musicali meno numerose rispetto a quelle dei centri più prestigiosi come Padova e Venezia, ma pur sempre in grado di svolgere attività musicali durante le celebrazioni dell'intero anno liturgico. ${ }^{14}$

Il secondo libro de mottetti a una e due voci, stampato presso l'erede di Angelo Gardano nel 1612, è dedicato al non altrimenti noto Giulio Gallo, "cittadino" della Magnifica Comunità di Monselice. ${ }^{15}$ La raccolta, conservata in un'unica copia presso la Westminster Abbey Library a Londra, contiene diciotto composizioni: quattro a voce sola, nove a due voci e cinque "Salmi che si cantano alle Compiete" per due tenori e basso, tutte sostenute dal basso continuo. In linea con molte altre raccolte sacre dell'epoca, questa raccolta prevede l'impiego di diversi organici e dunque poteva rispondere anche alle esigenze delle cappelle musicali meno numerose, come potevano essere quelle di Monselice e Montagnana, presso le quali si trovò a operare lo stesso Gualtieri (tab. 2).

Per quanto riguarda i testi musicati nella raccolta di Gualtieri, otto sono biblici e due si possono reperire nell'Antifonale monastico; Franciscus pauper et humilis e Salve sancte Pater sono dedicati al santo di Assisi, mentre i restanti mottetti impiegano testi religiosi o devozionali in prosa latina.

${ }^{12}$ Con i quarantanove mottetti a voce sola, stampati nelle sue raccolte del 1610 e 1615, Barbarino risulta sicuramente essere, tra i musicisti attivi in ambito padovano, uno dei più prolifici compositori di mottetti monodici.

${ }^{13}$ RISM $1625^{2}$.

${ }^{14}$ Lovato, "Musica e liturgia", 231-249; Scattolin, "La cappella musicale".

${ }^{15}$ La dedica non fornisce elementi che possano consentire l'identificazione del dedicatario o chiarire le circostanze per le quali la collezione venne composta. 
Tabella 2

Antonio Gualtieri, Il secondo libro de mottetti a una e due voci (1612): incipit dei testi e organico

\begin{tabular}{|c|c|c|}
\hline Incipit dei testi & Organico & Provenienza \\
\hline 1. Assumpta est Maria & A ebc & \\
\hline 2. Egredimini et videte filiae & A ebc & Cantico dei cantici, 3,11 \\
\hline 3. Confirma hoc Deus & Sebc & Ps. $67,29-30$ \\
\hline 4. Adiuro vos filiae & Tebc & \\
\hline 5. Angelus ad pastores ait & $2 \mathrm{Tebc}$ & Ant. In Nativitate Domini \\
\hline 6. Consolamini pastores quia hodie & $2 \mathrm{Tebc}$ & \\
\hline 7. Veni pulchritudo animae & $2 \mathrm{Sebc}$ & \\
\hline 8. Franciscus pauper et humilis & $2 \mathrm{Tebc}$ & \\
\hline 9. Salve sancte Pater & $2 \mathrm{Tebc}$ & Inno per le Stimmate di San Francesco \\
\hline 10. Carole maiorum generosa & $2 \mathrm{Tebc}$ & \\
\hline 11. Audi Domine hymnum & S, Tebc & III Libro dei Re, 8, 28-29 \\
\hline 12. Iubilemus omnes et diem & $2 \mathrm{Tebc}$ & \\
\hline 13. Estote fortes in bello & T, B ebc & Ant. Commune Apostolorum et Evangelistarum (extra tempus paschale) \\
\hline 14. Cum invocarem exaudivit & $2 \mathrm{~T}, \mathrm{~B} \mathrm{ebc}$ & Ps. $4,2,3,5,7,9$ \\
\hline 15. In te Domine speravi & $2 \mathrm{~T}, \mathrm{~B} \mathrm{ebc}$ & Ps. $30,2,3,5$ \\
\hline 16. Qui habitat in adiutorio & $2 \mathrm{~T}, \mathrm{~B} \mathrm{ebc}$ & Ps. $90,1,3,5,7,9,11,13,15$ \\
\hline 17. Ecce nunc benedicite Dominum & $2 \mathrm{~T}, \mathrm{~B} \mathrm{e} \mathrm{bc}$ & Ps. $133,1-2$ \\
\hline 18. Nunc dimittis servum tuum & $2 \mathrm{~T}, \mathrm{~B} \mathrm{ebc}$ & Lc, 2, 29, 31 \\
\hline
\end{tabular}

Dal punto di vista della scrittura musicale, le figure ritmiche utilizzate vanno dalla semicroma alla longa, come possiamo riscontrare anche nel Parnassus, al cui interno, tuttavia, le ricche diminuzioni sono ulteriormente impreziosite dall'utilizzo delle biscrome. In linea generale possiamo affermare che nei mottetti di Gualtieri i passaggi particolarmente diminuiti sono riservati ai mottetti a voce sola, mentre le composizioni a due o più voci prevedono una scrittura meno virtuosistica. All'interno del Parnassus, invece, anche le composizioni a più voci presentano diminuzioni più numerose ed elaborate (si veda, ad esempio, la lunga successione di semicrome in corrispondenza della parola "aromata", posta in chiusura del mottetto Veni in hortum di Giacomo Filippo Biumi). ${ }^{16}$

Come dimostra l'esempio seguente, Gualtieri generalmente annota per esteso tutte le diminuzioni che un cantante dell'epoca avrebbe probabilmente eseguito comunque (es. mus. 1a). Epurata la linea dalle diminuzioni, risulta la seguente struttura melodica basilare che, pur nella sua essenziale semplicità, si rivela comunque compiuta (es. mus. 1b).

\section{Esempio musicale 1a}

Antonio Gualtieri, Assumpta est Maria, bb. 38-39

A

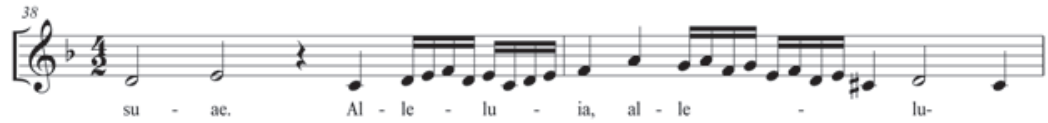

${ }^{16}$ Antonicek, Parnassus musicus Ferdinandaeus, 94 (bb. 112-117). 


\section{Esempio musicale 1b}

Antonio Gualtieri, Assumpta est Maria, bb. 38-39 senza diminuzioni

A

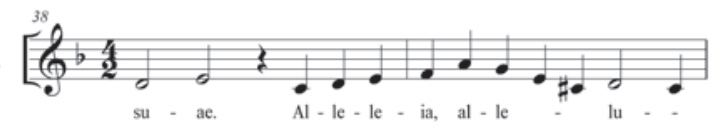

Si tratta di un procedimento comune a molti compositori dell'epoca, ed è probabile che qualunque cantore professionista, di fronte a una simile struttura semplificata, avrebbe comunque introdotto una o più diminuzioni per arricchire e variare la linea della melodia, specialmente in corrispondenza della parola alleluja. Lo stesso ribattuto di gola indicato dalla successione di quattro semicrome (cfr. l'esempio seguente) dimostra come Gualtieri fosse solito indicare in maniera pedissequa alcuni dettagli musicali di uso comune, la cui esplicitazione si sarebbe potuta omettere (es. mus. 2). Anche in diversi mottetti presenti nel Parnassus troviamo indicate in extenso diminuzioni afferenti alla sfera della prassi, come accade nel mottetto Cantate Domino di Monteverdi (es. mus. 3). ${ }^{17}$

\section{Esempio musicale 2}

Antonio Gualtieri, Assumpta est Maria, bb. 26-28

A

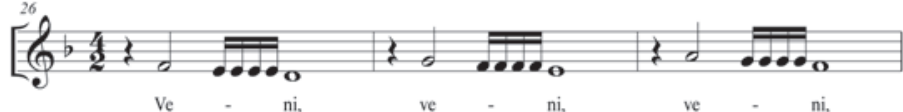

\section{Esempio musicale 3}

Claudio Monteverdi, Cantate Domino, bb. 117-119

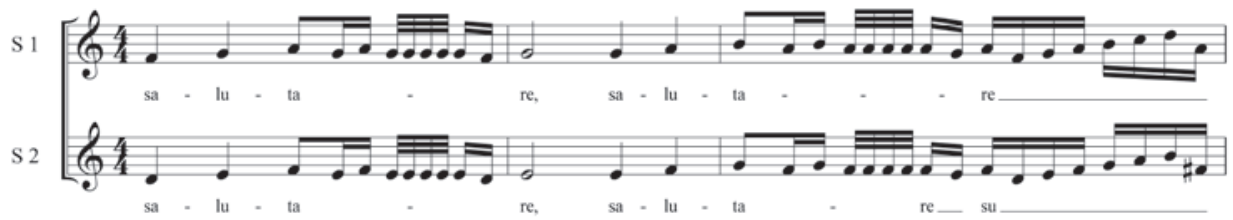

È probabile che la raccolta di Gualtieri fosse destinata a cantanti poco più che amatoriali, o comunque poco avvezzi a introdurre adeguate diminuzioni, a meno che non se le trovassero scritte. In alternativa, il compositore potrebbe aver voluto indicare la precisa modalità di esecuzione della sua musica, manifestando la medesima consapevolezza espressa da Lodovico da Viadana negli avvertimenti "a' benigni lettori" apposti ai suoi Cento concerti ecclesiastici. ${ }^{18}$

Per quanto riguarda l'aspetto mensurale, come nei mottetti del Parnassus, anche nella raccolta di Gualtieri il tactus comunemente impiegato è il tempus imperfectum non

${ }^{17}$ Ibid., 84-90.

18 "Et prima, che questa sorte di concerti deve cantarsi gentilmente con discrettione et leggiadria, usando gli acenti con raggione et passaggi con misura et a' suoi lochi, sovra tutto non aggiungendo alcuna cosa più di quello che in loro si ritrova stampato; percioché vi sono talhora certi cantanti i quali, perché si trovano favoriti dalla natura d'un poco di garante, mai cantano nella maniera che fanno i canti, non si accorgendo essi che hoggidì questi tali non sono grati, anzi sono pochissimo stimati particolarmente in Roma, dove fiorisce la vera professione del cantar bene". Viadana, "Concerti", prefazione. 
diminutum, tranne per il mottetto Iubilemus omnes et diem composto in tempus perfectum diminutum. ${ }^{19}$ Se si eccettuano cinque mottetti (Egredimini et videte filiae, Veni pulchritudo animae, Franciscus pauper et humilis, Salve sancte Pater e Audi Domine hymnum), gli altri hanno una o più sezioni in tempo ternario notate in 3 o in 3/2. Questi episodi assumono funzioni diverse e servono per descrivere le parole che invitano al canto e alla gioia, come "gaudent angeli", "alleluia" e "iubilemus omnes", oppure evidenziano incisi testuali dal significato pregnante quali possono essere, a titolo esemplificativo, "semper benedicite" e "et glorificabo eum". In altre situazioni il ritmo ternario ha la funzione di enfatizzare ulteriormente le espressioni di esortazione presenti nel testo ("dicite", "pugnate cum antiquo serpente", "irascimini"). Tra i quattordici mottetti del Parnassus presi in cosiderazione, sette composizioni presentano una o più sezioni in tempo ternario, mentre i restanti protraggono il tempus imperfectum per l'intera composizione. Una caratteristica ricorrente della scrittura di Gualtieri che possiamo riscontrare anche in due mottetti del Parnassus è la riproposizione di intere sezioni - generalmente in mensura ternaria e in corrispondenza delle parole di giubilo - ripetute identiche all'interno della composizione (creando l'effetto tipico dell'epiphora). ${ }^{20}$

Osservando la disposizione delle chiavi e l'estensione delle singole parti nella raccolta di Gualtieri (tab. 3) e nel Parnassus (tab. 4), si nota che quando i mottetti sono destinati a due voci dello stesso registro la tessitura è la medesima, per cui c'è una totale omogeneità tra le due linee che si alternano e si intersecano sullo stesso piano vocale. Negli altri casi la tessitura rispetta la normale estensione del timbro vocale prescelto, per cui non ci sono linee che si estendono fino all'estremo acuto o grave. Fa eccezione il mottetto Ecce quam bonum di Giovanni Sansoni, in cui il basso secondo si spinge fino al $\mathrm{do}_{1}{ }^{21}$

Tabella 3

A. Gualtieri, Il secondo libro de mottetti a una e due voci (1612): chiavi ed estensioni vocali

\begin{tabular}{|l|l|l|l|l|l|l|l|l|l|}
\hline Incipit dei testi & \multicolumn{2}{l|l}{ Canto } & \multicolumn{2}{l|l}{ Alto } & \multicolumn{2}{l|}{ Basso } \\
\hline 1. Assumpta est Maria & & & $\mathrm{Do}^{3}$ & $\mathrm{Si}_{2}-\mathrm{la}_{3}$ & & & & \\
\hline 2. Egredimini et videte filiae & & & $\mathrm{Do}^{3}$ & $\mathrm{Si}_{2}-\mathrm{la}_{3}$ & & & & \\
\hline 3. Confirma hoc Deus & $\mathrm{Vl}$ & $\mathrm{fa}_{3}-\mathrm{Sol}_{4}$ & & & & & & \\
\hline 4. Adiuro vos filiae & & & & & $\mathrm{Do}^{4}$ & $\mathrm{fa}_{2}-\mathrm{re}_{3}$ & & \\
\hline 5. Angelus ad pastores ait & & & $\mathrm{Do}^{4}$ & $\mathrm{sol}_{2}-\mathrm{la}_{3}$ & $\mathrm{Do}^{4}$ & $\mathrm{sol}_{2}-\mathrm{la}_{3}$ & & \\
\hline 6. Consolamini pastores quia hodie & & & $\mathrm{Do}^{4}$ & $\mathrm{do}_{2}-\mathrm{re}_{3}$ & $\mathrm{Do}^{4}$ & $\mathrm{do}_{2}-\mathrm{re}_{3}$ & & \\
\hline 7. Veni pulchritudo animae & $\mathrm{Do}^{1}$ & $\mathrm{mi}_{3}-\mathrm{mi}_{4}$ & $\mathrm{Do}^{1}$ & $\mathrm{mi}_{3}-\mathrm{mi}_{4}$ & & & & \\
\hline 8. Franciscus pauper et humilis & & & $\mathrm{Do}^{4}$ & $\mathrm{do}_{2}-\mathrm{mi}_{3}$ & $\mathrm{Do}^{4}$ & $\mathrm{re}_{2}-\mathrm{mi}_{3}$ & & \\
\hline 9. Salve sancte pater & & & $\mathrm{Do}^{4}$ & $\mathrm{re}_{2}-\mathrm{mi}_{3}$ & $\mathrm{Do}^{4}$ & $\mathrm{do}_{2}-\mathrm{re}_{3}$ & & \\
\hline 10. Carole maiorum generosa & & & $\mathrm{Do}^{4}$ & $\mathrm{re}_{2}-\mathrm{mi}_{3}$ & $\mathrm{Do}^{4}$ & $\mathrm{re}_{2}-\mathrm{mi}_{3}$ & & \\
\hline 11. Audi Domine hymnum & $\mathrm{Vl}$ & $\mathrm{sol}_{3}-\mathrm{sol}_{4}$ & & & $\mathrm{Do}^{3}$ & $\mathrm{sol}_{2}-\mathrm{la}_{3}$ & & \\
\hline
\end{tabular}

${ }^{19}$ Dei quattordici mottetti del Parnassus presi in considerazione soltanto il mottetto Cantate Domino di Claudio Monteverdi presenta un incipit in tempus perfectum diminutum.

${ }^{20}$ Antonicek, Parnassus musicus Ferdinandaeus, 9-11 e 84-91; Gualtieri, "Il secondo libro", n. 3, 4, 6 e 12 .

${ }^{21}$ Antonicek, Parnassus musicus Ferdinandaeus, 107-110. 


\begin{tabular}{|l|l|l|l|l|l|l|l|l|}
\hline Incipit dei testi & \multicolumn{2}{l|}{ Canto } & \multicolumn{2}{l|}{ Alto } & \multicolumn{2}{l|}{ Tenore } & \multicolumn{2}{l|}{ Basso } \\
\hline 12. Iubilemus omnes et diem & & & $\mathrm{Do}^{3}$ & $\mathrm{fa}_{2}-\mathrm{la}_{3}$ & $\mathrm{Do}^{3}$ & $\mathrm{sol}_{2}-\mathrm{la}_{3}$ & & \\
\hline 13. Estote fortes in bello & & & & & $\mathrm{Do}^{4}$ & $\mathrm{re}_{2}-\mathrm{mi}_{3}$ & $\mathrm{Fa}^{4}$ & $\mathrm{sol}_{1}-\mathrm{do}_{3}$ \\
\hline 14. Cum invocarem exaudivit & & & $\mathrm{Do}^{4}$ & $\mathrm{do}_{2}-\mathrm{mi}_{4}$ & $\mathrm{Do}^{4}$ & $\mathrm{do}_{2}-\mathrm{mi}_{3}$ & $\mathrm{Fa}^{4}$ & $\mathrm{sol}_{1}-\mathrm{la}_{2}$ \\
\hline 15. In te Domine speravi & & & $\mathrm{Do}^{3}$ & $\mathrm{fa}_{2}-\mathrm{la}_{3}$ & $\mathrm{Do}^{3}$ & $\mathrm{fa}_{2}-\mathrm{la}_{3}$ & $\mathrm{Fa}^{3}$ & $\mathrm{sol}_{1}-\mathrm{re}_{3}$ \\
\hline 16. Qui habitat in adiutorio & & & $\mathrm{Do}^{4}$ & $\mathrm{do}_{2}-\mathrm{re}_{3}$ & $\mathrm{Do}^{4}$ & $\mathrm{do}_{2}-\mathrm{re}_{3}$ & $\mathrm{Fa}^{4}$ & $\mathrm{fa}_{1}-\mathrm{si}_{2}$ \\
\hline 17. Ecce nunc benedicite Dominum & & & $\mathrm{Do}^{3}$ & $\mathrm{sol}_{2}-\mathrm{la}_{3}$ & $\mathrm{Do}^{3}$ & $\mathrm{fa}_{2}-\mathrm{la}_{3}$ & $\mathrm{Fa}^{4}$ & $\mathrm{si}_{1}-\mathrm{re}_{3}$ \\
\hline 18. Nunc dimittis servum tuum & & & $\mathrm{Do}^{4}$ & $\mathrm{mi}_{2}-\mathrm{mi}_{3}$ & $\mathrm{Do}^{4}$ & $\mathrm{mi}_{2}-\mathrm{mi}_{3}$ & $\mathrm{Fa}^{4}$ & $\mathrm{sol}_{1}-\mathrm{sol}_{2}$ \\
\hline
\end{tabular}

\section{Tabella 4}

Parnassus, Mottetti a una voce e due voci pari, estensione

\begin{tabular}{|l|c|c|c|c|c|c|}
\hline Mottetto & Voce & Chiave & Esten-sione & Voce & Chiave & Esten-sione \\
\hline Pellegrini, Dominus regitme & $\mathrm{C}$ & $\mathrm{Do}^{1}$ & $\mathrm{re}_{3}-\mathrm{mi}_{4}$ & & & \\
\hline Priuli, Egredimini filiae Sion & $\mathrm{C}$ & $\mathrm{Do}^{1}$ & $\mathrm{Re}_{3}-\mathrm{mi}_{4}$ & & & \\
\hline Valentini, Benedicam Dominum & $\mathrm{B}$ & $\mathrm{Fa}^{4}$ & $\mathrm{fa}_{1}-\mathrm{do}_{3}$ & & & \\
\hline Cavaccio, Paratum cor meum & $\mathrm{T}$ & $\mathrm{Do}^{4}$ & $\mathrm{mi}_{2}-\mathrm{fa}_{3}$ & & & \\
\hline Barbarini, Omnes sitientes & $\mathrm{T}$ & $\mathrm{Do}^{4}$ & $\mathrm{re}_{2}-\mathrm{fa}_{3}$ & & & \\
\hline Priuli, Adoramus te Domine & $\mathrm{A}$ & $\mathrm{Do}^{3}$ & $\mathrm{mi}_{2}-\mathrm{la}_{3}$ & & & \\
\hline Turini, Sacrificemus Reginae Caeli & $\mathrm{A}$ & $\mathrm{Do}^{3}$ & $\mathrm{fa}_{2}-\mathrm{la}_{3}$ & & & \\
\hline Corradini, Attende Domine & $\mathrm{C}$ & $\mathrm{Do}^{1}$ & $\mathrm{do}_{3}-\mathrm{fa}_{4}$ & & & \\
\hline Monteverdi, Cantate Domino & $\mathrm{CI}$ & $\mathrm{Do}^{1}$ & $\mathrm{do}_{3}-\mathrm{fa}_{4}$ & $\mathrm{CII}$ & $\mathrm{Do}^{1}$ & $\mathrm{si}_{3}-\mathrm{fa}_{4}$ \\
\hline Biumi, Veni in hortum & $\mathrm{CI}$ & $\mathrm{Do}^{1}$ & $\mathrm{do}_{3}-\mathrm{fa}_{4}$ & $\mathrm{CII}$ & $\mathrm{Do}^{1}$ & $\mathrm{do}_{3}-\mathrm{fa}_{4}$ \\
\hline Bontempi, Quam brevefestum & $\mathrm{TI}$ & $\mathrm{Do}^{4}$ & $\mathrm{do}_{2}-\mathrm{mi}_{3}$ & $\mathrm{TII}$ & $\mathrm{Do}^{4}$ & $\mathrm{do}_{2}-\mathrm{mi}_{3}$ \\
\hline Gabussi, Ocrux benedicta & $\mathrm{AI}$ & $\mathrm{Do}^{3}$ & $\mathrm{sol}_{2}-\mathrm{Si}_{3}$ & $\mathrm{AII}$ & $\mathrm{Do}^{3}$ & $\mathrm{sol}_{2}-\mathrm{Si}_{3}$ \\
\hline Sansoni, Ego dormio & $\mathrm{AI}$ & $\mathrm{Do}^{3}$ & $\mathrm{fa}_{2}-\mathrm{la}_{3}$ & $\mathrm{AII}$ & $\mathrm{Do}^{3}$ & $\mathrm{fa}_{2}-\mathrm{la}_{3}$ \\
\hline Sansoni, Ecce quam bonum & $\mathrm{BI}$ & $\mathrm{Fa}^{4}$ & $\mathrm{mi}_{1}-\mathrm{Si}_{2}$ & $\mathrm{BII}$ & $\mathrm{Fa}^{4}$ & $\mathrm{do}_{1}-\mathrm{la}_{2}$ \\
\hline
\end{tabular}

Nella raccolta di Gualtieri del 1612 compare, per la prima volta nelle sue opere, una parte di "basso per l'organo" con alcune indicazioni per la realizzazione armonica. Queste indicazioni non sono ancora così specifiche come quelle che si rilevano nella successiva raccolta di mottetti, nella quale viene indicata anche la cifratura del basso continuo (che in questa raccolta è assente e che possiamo trovare nella parte del basso di alcuni mottetti inclusi nel Parnassus). Nelle composizioni di Gualtieri, così come in quelle della raccolta dedicata all'arciduca Ferdinando, la principale funzione del basso per l'organo è quella di accompagnamento e sostegno armonico alla linea o alle linee vocali. Infatti la parte affidata allo strumento è prevalentemente "seguente", ossia riprende quella della voce più grave, formando una linea grave continua. In alcuni casi, più rari ma non così sporadici, il basso strumentale ha un ruolo concertante e imitativo, proponendo un dialogo, seppur breve, con una o più voci soliste.

Dal punto di vista armonico, la raccolta di Gualtieri non presenta una scrittura particolarmente ardita, anche se alcuni passaggi sono resi interessanti dall'uso del ritardo o dell'anticipo di un'armonia. Caratteristici risultano alcuni rari intervalli di seconda aumentata, come accade nella voce del Tenore secondo nel mottetto Franciscus pauper 
et humilis, non comuni nella pratica compositiva e, proprio per questo, particolarmente adatti a rendere gli incisi maggiormente interessanti (es. mus. 4).

\section{Esempio musicae 4}

Antonio Gualtieri, Franciscus pauper et humilis, bb. 32-34

$\mathrm{T} 1$

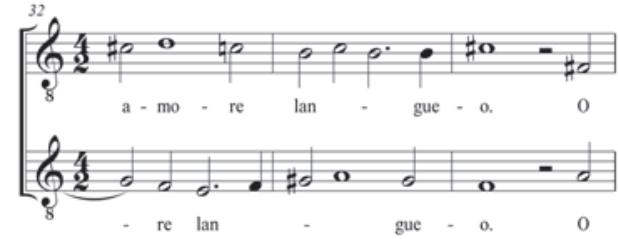

In sede di cadenza finale prevale la cadenza perfetta (V-I), mentre la cadenza plagale (IV-I) viene impiegata solo in tre casi. È abbastanza frequente il ricorso a procedimenti di progressione, che rendono incalzante l'andamento melodico. Questa preferenza in sede di cadenza è confermata anche dai quattordici mottetti del Parnassus oggetto del nostro confronto, fra cui soltanto Veni in hortum meum di Giacomo Filippo Biumi impiega la cadenza plagale, mentre gli altri tredici ricorrono alla cadenza perfetta. ${ }^{22}$

È particolarmente interessante notare l'attenzione che i compositori prestano al rapporto tra testo e musica. Per sottolineare determinate parole o frasi del testo, Gualtieri, come molti altri compositori, ricorre all'impiego di diminuzioni, al trattamento omoritmico delle voci e all'espediente dell'interruptio. In altre occasioni, invece, i termini vengono resi musicalmente grazie al ricorso a figure retoriche. Ad esempio, parole come "assumpta" e "coelum" (es. mus. 5) sono rese con un moto ascendente della melodia (anabasis), espediente che possiamo trovare anche in corrispondenza della parola "surrexi" nel mottetto Ego dormio di Giovanni Sansoni. In questo caso la parola è descritta attraverso una linea melodica caratterizzata dall'intervallo di quarta ascendente e che porta l'alto secondo all'apice melodico dell'intera composizione. ${ }^{23}$

All'opposto, il verbo "cadent" (es. mus. 6) è descritto da tutte le voci con un intervallo di ottava discendente, generando l'effetto di katabasis. La stessa cosa avviene anche nel mottetto Ecce quam bonum di Giovanni Sansoni in corrispondenza del verbo "descendit", valorizzato e reso ancora più pregnante dal ritmo puntato e dal raggiungimento delle due note più gravi della composizione, $\mathrm{re}_{1} \mathrm{e} \mathrm{do}_{1} \cdot{ }^{24}$

\section{Esempio musicale 5}

Antonio Gualtieri, Assumpta est Maria, bb. 1-5

A

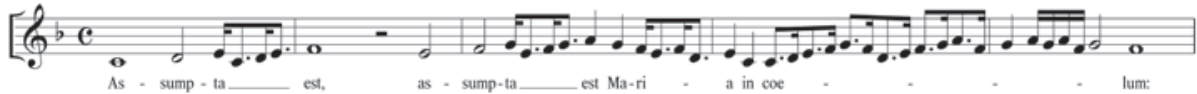

${ }^{22}$ Ibid., 91-94.

${ }^{23}$ Ibid., 76-77, bb. 52-57.

${ }^{24}$ Ibid., 107-110, bb. 34-62. 


\section{Esempio musicale 6}

Antonio Gualtieri, Qui habitat in adiutorio, bb. 29-30

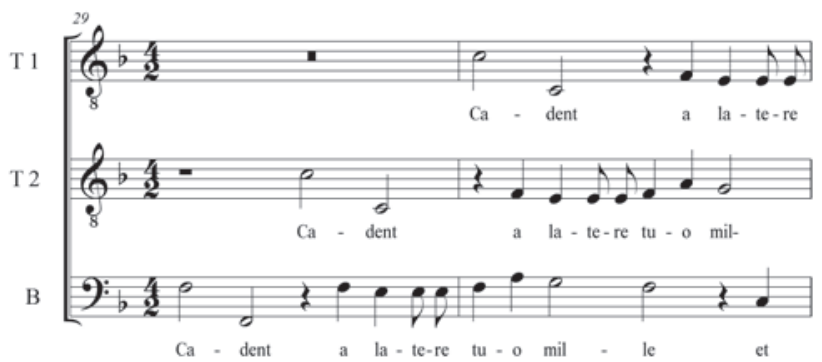

Frequente, all'interno della raccolta di Gualtieri, è l'uso di due figure retoriche simili, caratterizzate dall'iterazione di formule melodiche o dalla ripetizioni di frasi melodiche: l'anafora o repetitio, cioè la ripetizione di uno stesso inciso alla stessa altezza, e il poliptoton, ossia la ripetizione della stessa frase melodica nelle diverse voci. Tra i molteplici casi di utilizzo di queste figure retoriche nell'esempio seguente è possibile incontrare le due figure retoriche all'interno dello stesso segmento musicale (es. mus. 7).

\section{Esempio musicale 7}

Antonio Gualtieri, Qui habitat in adiutorio, bb. 36-38

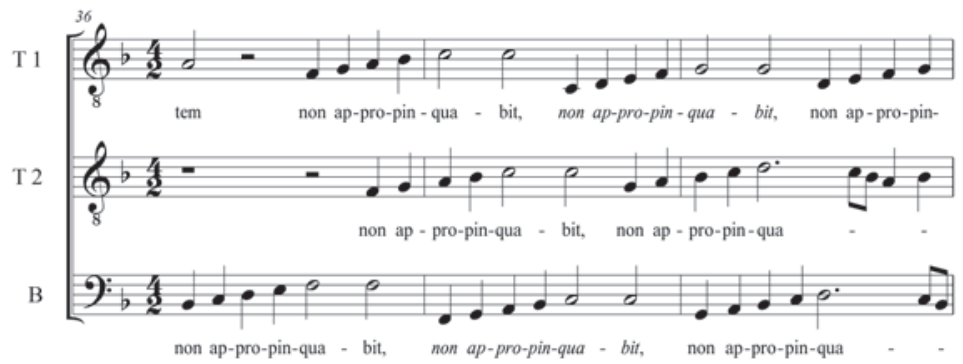

Anche l'interruptio ricorre spesso in questa raccolta di mottetti, dove viene utilizzata per separare le battute finali dal resto della composizione, oppure per porre in evidenza determinati incisi testuali grazie a una pausa che coinvolge tutte le voci. In ogni caso si tratta di un espediente che il compositore adopera per richiamare l'attenzione su un frammento testuale che altrimenti rischierebbe di passare inosservato.

Vi sono alcuni procedimenti compositivi che Gualtieri utilizza soltanto nei mottetti a voce sola, come l'uso ricorrente della figurazione ritmica Semicroma-Croma puntata, ossia il cosiddetto ritmo 'lombardo' o 'alla zoppa' tanto caro sia a Claudio Monteverdi, sia a molti compositori del primo Seicento. Questo ritmo irregolare che, proprio per la sua atipicità, riesce a dare vivacità alla frase, ritorna infatti anche in diverse composizioni a voce sola o a poche voci e basso continuo dei maestri attivi nel territorio padovano e veneziano durante la prima metà del Seicento, trovando ampio uso anche all'interno della silloge dedicata all'arciduca Ferdinando.

All'interno della raccolta di Gualtieri merita particolare attenzione il mottetto Adiuro vos filiae, introdotto dalla prescrizione "Tenore voce sola in echo", in cui le ultime sillabe di alcune parole vengono puntualmente ripetute in forma di risposta sonora. La scrittura 
in eco non era certo insolita tra le raccolte del primo Seicento, e lo stesso Gualtieri aveva già utilizzato questa tecnica nel mottetto a otto voci Confitemini Domino. In Adiuro vos l'aspetto più significativo è, però, la presenza di indicazioni dinamiche, per cui ogni sillaba in eco viene indicata con la lettera "P" ( e doveva quindi esser eseguita piano), mentre la frase che segue è indicata con la lettera "F", per suggerire un aumento dell'intensità. Le indicazioni diventano ancora più specifiche a conclusione del brano, prescrivendo la realizzazione delle ultime note in decrescendo, dal piano ("P") al pianissimo (indicato come "P.P." e talvolta anche come "PPP."). ${ }^{25}$

Altre peculiarità sono invece proprie dei nove mottetti a due voci e basso continuo, che hanno una costruzione simile tra loro. Ad eccezione dei mottetti Salve sancte Pater, in cui le due voci intonano la parola "salve" con valori lati procedendo per terze, ed Estote fortes in bello, dove l'imitazione non è rigorosa come negli altri casi, nella maggior parte dei casi l'incipit è affidato a una voce che propone il tema, successivamente riproposto dall'altra (es. mus. 8). Lo stesso procedimento è riscontrabile nei mottetti a due voci e basso continuo del Parnassus. L'omoritmia viene utilizzata principalmente per mettere in evidenza o per enfatizzare un determinato inciso testuale. Molto frequente è la contrapposizione tra sezioni omoritmiche ed episodi in contrappunto per descrivere particolari concetti descritti nel testo.

\section{Esempio musicale 8}

Antonio Gualtieri, Carole maiorum generosa, bb. 1-4

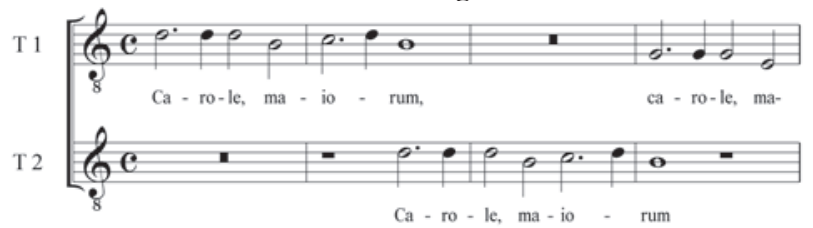

Per concludere, un breve accenno agli ultimi cinque mottetti della raccolta di Gualtieri, i "Salmi che si cantano alle Compiete", che si differenziano marcatamente dagli altri. Innanzitutto l'organico è composto da tre voci maschili ( $\mathrm{T} 1, \mathrm{~T} 2, \mathrm{~B})$ accompagnate da un basso continuo e la linea vocale più grave ha una funzione di sostegno, confermata dal fatto che corrisponde, quasi sempre esattamente, alla linea dell'organo. La differenza più notevole, però, sta nel tipo di scrittura, che in questa sezione è caratterizzata da un andamento prevalentemente accordale e omoritmico. Alcuni brevi spunti imitativi e sezioni in mensura ternaria introducono qualche elemento di varietà, mentre sono del tutto assenti le ricche diminuzioni dei mottetti a voce sola. Pertanto, queste composizioni presentano un carattere più corale rispetto agli altri mottetti, chiaramente destinati a un'esecuzione solistica; inoltre, sebbene nel Salmo 90 alcuni versetti siano affidati a una voce solista, la

${ }^{25}$ L'indicazione proposta da Gualtieri di eseguire una frase piano o forte si trova impiegata già alla fine del Cinquecento per segnalare un effetto di eco, ad esempio in alcune composizioni del Primo libro delle villanelle (1596) di Aurelio Bonelli. La stessa indicazione veniva utilizzata per segnalare l'alternanza tra due cori o gruppi strumentali, come nella Sonata pian e forte di Giovanni Gabrieli. Nella produzione di Gualtieri, tuttavia, il mottetto Adiuro vos filiae è l'unica composizione in cui compaiono simili indicazioni dinamiche. 
scrittura rimane coerente con il resto della composizione essendo priva di diminuzioni o passaggi particolarmente arditi.

Ad eccezione del cantico Nunc dimittis servum tuum, i quattro salmi conclusivi, come di consueto, sono introdotti dall'intonazione gregoriana. Anzi, poiché Gualtieri intona soltanto alcuni versetti dispari dei salmi, ${ }^{26}$ è probabile che la loro esecuzione avvenisse secondo la pratica altrettanto tradizionale e diffusa di alternare sezioni polifoniche ad altre in canto piano o con l'organo. Questa raccolta, infatti, è stata pubblicata nel 1612, quando Gualtieri era maestro di cappella nella collegiata di Monselice, dove il canto monodico costituiva il compito principale del capitolo obbligato alla liturgia corale. ${ }^{27}$

In conclusione possiamo affermare che Antonio Gualtieri, un autore sinora poco conosciuto, seppe far proprie le nuove tecniche compositive aggiornando la sua scrittura musicale. Il confronto fra le composizioni incluse nel suo primo libro di mottetti a una e due voci e continuo, pubblicato nel 1612, e le composizioni di diversi autori dotate di un medesimo organico incluse nel Parnassus, pubblicato soltanto tre anni dopo, ha infatti mostrato come la scrittura di Gualtieri si riveli del tutto in linea con le principali caratteristiche di composizioni prodotte in altre aree della penisola e importate nella cappella musicale di Graz.

\section{Appendice 1}

Raccolte individuali dei compositori attivi a Padova nei primi quindici anni del Seicento.

\begin{tabular}{|l|l|l|}
\hline Anno & Titolo dell'opera & \multicolumn{1}{l|}{ Città, editore } \\
\hline COSTANZO PORTA (RISM P 5171-5190) & Venezia, Antonio Gardano \\
\hline 1555 & Motectorum [...] liber primus, quinque vocibus. & Venezia, Antonio Gardano \\
\hline 1555 & Il primo libro de madrigali a quattro voci. & Venezia, Antonio Gardano \\
\hline 1559 & Liber primus motectorum quatuor vocum. & Venezia, Antonio Gardano \\
\hline 1559 & Liber primus motectorum quatuor vocum. & Venezia, Antonio Gardano \\
\hline 1565 & $\begin{array}{l}\text { Il primo libro di madrigali a cinque voci. } \\
\text { (RISM PP 5173a) }\end{array}$ & Venezia, Francesco Rampazetto \\
\hline $1566 \mathrm{a}$ & $\begin{array}{l}\text { Quinque vocum musica in introiutus missarum, quae in diebus } \\
\text { dominicis toto anno celebrantur, iuxta morem Sanctae Romanae } \\
\text { Ecclesiae. }\end{array}$ & $\begin{array}{l}\text { Venezia, Claudio Correggio e Fausto } \\
\text { Betanio }\end{array}$ \\
\hline $1588^{2} \mathrm{a}$ & {$[\ldots]$ nunc ab auctore diligentissime emendata. } & Venezia, Angelo Gardano \\
\hline
\end{tabular}

${ }^{26}$ Fa eccezione solo il mottetto Ecce nunc benedicite, in cui Gualtieri mette in musica solamente i primi due versetti del salmo 133, prevedendo l'intonazione gregoriana soltanto per l'emistichio iniziale.

${ }^{27}$ Lovato, "Musica e liturgia", 231-249. 
Chiara Comparin: Il secondo libro de mottetti a una e due voci di Antonio Gualtieri e il Parnassus musicus Ferdinandaeus

\begin{tabular}{|c|c|c|}
\hline Anno & Titolo dell'opera & Città, editore \\
\hline 1566b & $\begin{array}{l}\text { Quinque vocum musica in introitus missarum, quae in } \\
\text { solemnitatibus sanctorum omnium toto anno celebrantur, iuxta } \\
\text { morem Sanctae Romanae Ecclesiae. }\end{array}$ & $\begin{array}{l}\text { Venezia, Claudio Correggio e Fausto } \\
\text { Betanio }\end{array}$ \\
\hline $1588^{2} \mathrm{~b}$ & $\begin{array}{l}\text { Quinque vocum musica in introitus missarum, quae in } \\
\text { solemnitatibus sanctorum omnium toto anno celebrantur, iuxta } \\
\text { morem Sanctae Romanae Ecclesiae. }\end{array}$ & Venezia, Angelo Gardano \\
\hline 1569 & Il secondo libro de madrigali a cinque voci. & Venezia, Antonio Gardano \\
\hline 1571 & $\begin{array}{l}\text { Musica sex canenda vocibus, in nonnulla ex sacris litteris collecta } \\
\text { verba, liber primus. }\end{array}$ & Venezia, gli figliuoli di Antonio Gardano \\
\hline 1573 & Il terzo libro de madrigali a cinque voci. & Venezia, gli figliuoli di Antonio Gardano \\
\hline 1575 & $\begin{array}{l}\text { Litaniae Deiparae Virginis Mariae ex saxra scriptura } \\
\text { depromptae, quae in alma domo Lauretana omnibus diebus } \\
\text { sabbati,vigiliamrum, \& festorum eiusdem Beatae Virginis } \\
\text { decantari solent, cum musca octo vocum. }\end{array}$ & Venezia, Giorgio Angelieri \\
\hline 1578 & Missarum [a 4, 5 \& $6 \mathrm{v}]$ liber primus. & Venezia, Angelo Gardano \\
\hline 1580 & $\begin{array}{l}\text { Liber quinquaginta duorum motectorum, quatuor, quinque, sex, } \\
\text { septem, \& octo vocum. }\end{array}$ & Venezia, Angelo Gardano \\
\hline 1585 & $\begin{array}{l}\text { Musica sex canenda vocibus in nonnulla ex sacris litteris collecta } \\
\text { verba [...] liber tertius. }\end{array}$ & Venezia, Angelo Gardano \\
\hline 1586 & $\begin{array}{l}\text { Il quarto libro de madrigali a cinque voci, novamente da Marsilio } \\
\text { Cristofori raccolti }\end{array}$ & Venezia, Angelo Gardano \\
\hline 1602 & Hymnodia sacra totius per anni circulum quatuor vocibus. & Venezia, Angelo Gardano \\
\hline $1605 \mathrm{a}$ & Motectorum quinque vocum. & Venezia, Angelo Gardano \\
\hline $1605 b$ & $\begin{array}{l}\text { Psalmodia vespertina omnium solemnitatum octo vocibus } \\
\text { decantanda }[. . .] \text { cum quattuor canticis } B \text {. Virginis itidem octo } \\
\text { vocum, uno tantum excepto bis octo vocibus concinnendo. }\end{array}$ & Venezia, Angelo Gardano \\
\hline \multicolumn{3}{|c|}{ BARTOLOMEO RATTI (RISM R 324-328) } \\
\hline 1594 & $\begin{array}{l}\text { Cantiones in laudem Deiparae Virginis Mariae [...] liber primus, } \\
\text { cum quinque vocibus. }\end{array}$ & Venezia, Ricciardo Amadino \\
\hline 1594 & $\begin{array}{l}\text { Amorosi fiori colti in vago, a delitioso giardino, madrigali a } \\
\text { quatro voci con uno a otto in fine, composti in stil di canzonette. }\end{array}$ & Venezia, Ricciardo Amadino \\
\hline 1596 & $\begin{array}{l}\text { Ghirlanda de varii fiori amorosi. Secondo libro de madrigali a } \\
\text { quattro voci, composti in stil di canzonette, con un sonetto a otto } \\
\text { in fine, \& un dialogo a otto fatto nelle sontuose nozze dell' illustre } \\
\text { signor Giovan Martin Marchesi con l'illustre signora Lucina } \\
\text { Savorgnana. }\end{array}$ & Venezia, Ricciardo Amadino \\
\hline 1599 & Ardori amorosi, madrigali e canzonette a tre voci. & Venezia, Ricciardo Amadino \\
\hline 1605 & $\begin{array}{l}\text { Li brevi salmi intieri, che nelli vespri di tutte le solenità si cantano, } \\
\text { secondo il rito del sacro Concilio di Trento, a cinque voci con il } \\
\text { basso continuo per commodità de gl'organisti }\end{array}$ & Venezia, Ricciardo Amadino \\
\hline
\end{tabular}




\begin{tabular}{|c|c|c|}
\hline Anno & Titolo dell'opera & Città, editore \\
\hline \multicolumn{3}{|c|}{ GIULIO BELLI (RISM B 1745-1778) } \\
\hline 1584 & Canzonette [...] libro primo a quattro voci. & Venezia, Angelo Gardano \\
\hline $1586^{2}$ & Canzonette [...] libro primo a quattro voci. & Milano, Francesco \& eredi di Simon Tini \\
\hline $1595^{3}$ & [...] aggiontovi due canzonette nove. & Venezia, Angelo Gardano \\
\hline $\begin{array}{l}1586 \\
1597^{2} \\
1603 b^{3} \\
1604 a^{4}\end{array}$ & Missarum cum quinque vocibus liber primus. & Venezia, Angelo Gardano \\
\hline 1589 & Il primo libro de madrigali a cinque, et a sei voci. & Venezia, Angelo Gardano \\
\hline 1592 & Il secondo libro de madrigali a cinque et a sei voci. & Venezia, Ricciardo Amadino \\
\hline 1593 & $\begin{array}{l}\text { Il secondo libro delle canzonette a quatro voci }[. . .] \text { con alcune } \\
\text { romane a tre voci. }\end{array}$ & Venezia, Riccairdo Amadino \\
\hline 1593 & $\begin{array}{l}\text { Psalmi ad vesperas in totius anni solemnitatibus }[. . .] \text { quinque } \\
\text { vocibus [...] nunc primum in lucem editi } \\
\text { (RISM BB 1755a) }\end{array}$ & Venezia, Ricciardo Amadino \\
\hline $1598^{2}$ & $\begin{array}{l}\text { Psalmi ad vesperas in totius anni solemnitatibus [...] quinque } \\
\text { vocibus, duoque cantica B. Virginis, \& in fine addito Te Deum } \\
\text { laudamus, secunda impressio }\end{array}$ & Venezia, Ricciardo Amadino \\
\hline $1606^{3}$ & [...] tertia editio. & Venezia, Ricciardo Amadino \\
\hline 1595 & Missarum, sacrarumque cantionum octo vocibus liber primus. & Venezia, Ricciardo Amadino \\
\hline $1607^{2} \mathrm{c}$ & $\begin{array}{l}{[. .] \text { nunc denuo ab ipso autore recognitarum, ac etiamadditae }} \\
\text { partes infimae, ad beneplacitum organum pulsantis. }\end{array}$ & Venezia, Ricciardo Amadino \\
\hline & Basso generale per l'organo, delle messe et motetti a otto voci. & \\
\hline $1608^{3} \mathrm{~b}$ & & Venezia, Ricciardo Amadino \\
\hline 1596 & $\begin{array}{l}\text { Psalmi ad vesperas in totius anni solemnitatibus octo voc., } \\
\text { duoque cantica Beatae Virginis. }\end{array}$ & Venezia, Angelo Gardano \\
\hline $1600^{2} b$ & $\begin{array}{l}\text { Psalmi ad vesperas in totius anni solemnitatibus octo voc., } \\
\text { duoque cantica Beatae Virginis. }\end{array}$ & Venezia, Angelo Gardano \\
\hline 1607 & $\begin{array}{l}\text { Basso generale per l'organo dei salmi a otto voci. Che si cantano in } \\
\text { tutte le feste de l'anno. }\end{array}$ & Venezia, Angelo Gardano e fratelli \\
\hline $1615^{3}$ & $\begin{array}{l}\text { Psalmi ad vesperas in totius anni solemnitatibus octo vocibus, } \\
\text { duoque Cantica Beatae Virginis. Tertia impressione [...] con bc }\end{array}$ & $\begin{array}{l}\text { Venezia, stampa del Gardano, appresso } \\
\text { Bartolomeo Magni }\end{array}$ \\
\hline $1597^{2}$ & $\begin{array}{l}\text { Il primo libro de Madrigali a } 5 \text { et } 6 \text { voci novamente composte e } \\
\text { dato in luce }\end{array}$ & Venezia, Angelo Gardano \\
\hline
\end{tabular}


Chiara Comparin: Il secondo libro de mottetti a una e due voci di Antonio Gualtieri e il Parnassus musicus Ferdinandaeus

\begin{tabular}{|c|c|c|}
\hline Anno & Titolo dell'opera & Città, editore \\
\hline 1599 & Missarum quatuor vocibus liber primus. & Venezia, Angelo Gardano \\
\hline $1615^{2} b$ & $\begin{array}{l}{[. .] \text { \& missa pro defunctis, tertia impressione, cum basso }} \\
\text { continuato. }\end{array}$ & $\begin{array}{l}\text { Venezia, stampa del Gardano, appresso } \\
\text { Bartolomeo Magni }\end{array}$ \\
\hline $1622^{3}$ & [...] quarta impressione, cum basso continuo. & $\begin{array}{l}\text { Sub signo Gardani, appresso Bartolomeo } \\
\text { Magni }\end{array}$ \\
\hline $1600 \mathrm{a}$ & $\begin{array}{l}\text { Sacrarum cantionum quatuor, quinque, sex, octo et duodecim } \\
\text { vocibus cum litanijs Beatae Virginis Mariae, liber primus }\end{array}$ & Venezia, Angelo Gardano \\
\hline $1603 a$ & $\begin{array}{l}\text { Psalmi ad Vesperas in totius annifestivitatibus, ac tria cantica B. } \\
\text { Virginis Mariae, sex vocibus. }\end{array}$ & Venezia, Angelo Gardano \\
\hline $1604^{2} \mathrm{~b}$ & $\begin{array}{l}\text { Psalmi ad Vesperas in totius annifestivitatibus, ac tria cantica B. } \\
\text { Virginis Mariae, sex vocibus. }\end{array}$ & Venezia, Angelo Gardano \\
\hline $1607^{3} \mathrm{~d}$ & $\begin{array}{l}\text { Salmi vespertini a sei voci che si cantano in tutte le feste dell'anno } \\
{[\text { con bc]. }}\end{array}$ & Venezia, Angelo Gardano e fratelli \\
\hline 1605 & $\begin{array}{l}\text { Compieta, mottetti, \& letanie della Madonna a otto voci, falsi } \\
\text { bordoni sopra li otto toni a dui chori spezzati, con li Sicut erat } \\
\text { interi. }\end{array}$ & Venezia, Angelo Gardano \\
\hline $1607 a$ & $\begin{array}{l}\text { Compieta, falsi bordoni, mottetti, et litanie della Madonna a sei } \\
\text { voci, co'l basso generale per l'organo. }\end{array}$ & Venezia, Alessandro Raverii \\
\hline $1607 \mathrm{~b}$ & $\begin{array}{l}\text { Compieta, falsi bordoni, antifone, et litanie della Madonna a } \\
\text { quattro voci, co'l basso generale per l'organo [...] primo choro. } \\
{[\ldots] \text { a cinque voci }[. . .] \text { secondo choro. }}\end{array}$ & Venezia, Alessandro Raverii \\
\hline $1608 \mathrm{a}$ & $\begin{array}{l}\text { Missae sacrae quae cum quatuor, quinque, sex et octo vocibus } \\
\text { concinuntur, cum basso generali per organo. }\end{array}$ & Venezia, Angelo Gardano et fratelli \\
\hline $1613^{2} \mathrm{~b}$ & $\begin{array}{l}\text { Missae sacrae quae cum quatuor, quinque, sex et octo vocibus } \\
\text { concinuntur, cum basso generali per organo. }\end{array}$ & Aere Bartolomei Magni \\
\hline $1613 a$ & Concerti ecclesiastici a due et a tre voci & Venezia, Bartolomeo Magni \\
\hline $1621^{2}$ & Concerti ecclesiastici a due et a tre voci & Frankfurt, Nikolaus Stein \\
\hline 1622 & $\begin{array}{l}\text { Concerti ecclesiastici binis et trinis vocibus, cum basso continuo } \\
\text { ad organum } \\
\text { (RISM BB 1772a) }\end{array}$ & Antwerpen, Pierre Phalèse \\
\hline \multicolumn{3}{|c|}{ LUIGI BALBI (RISM B 748-749) } \\
\hline 1606 & $\begin{array}{l}\text { Ecclesiastici concentus canendi una, duabus, tribus \& quatuor } \\
\text { vocibus, aut organo, aut alijs quibusvis instrumentis eiusdem } \\
\text { generis, [...] liber primus. }\end{array}$ & Venezia, Alessandro Raverii \\
\hline 1606 & $\begin{array}{l}\text { Partitura delli concerti ecclesiastici a una, doi, tre, quattro, } \\
\text { cinque, sette et otto voci, per sonare nell'organo o altri } \\
\text { instrumenti con una canzone a quattro [...] libro primo. }\end{array}$ & Venezia, Alessandro Raverii \\
\hline
\end{tabular}




\begin{tabular}{|l|l|l|}
\hline Anno & Titolo dell'opera & Città, editore \\
\hline LELIO BERTANI (RISM B 2114-2116) & Brescia, Pietro Maria Marchetti \\
\hline 1584 & Il primo libro de madrigali a cinque voci. & Venezia, Angelo Gardano \\
\hline 1585 & Il primo libro de' madrigali a sei voci & Venezia, Angelo Gardano e fratelli \\
1607 & & Brescia, Vincenzo Sabbio \\
\hline 1585 & Madrigali spirituali a tre voci (manca in RISM) & \\
\hline BARTOLOMEO FAVARETTO (RISM F 149) & Venezia, Giacomo Vincenti \\
\hline 1604 & $\begin{array}{l}\text { Laude spirituali a quattro voci nella Assomptione della gloriosa } \\
\text { Vergine Maria }\end{array}$ \\
\hline
\end{tabular}

\section{Appendice 2}

Raccolte stampate tra il 1600 e il 1615 che includono composizioni di maestri attivi a Padova nei primi quindici anni del Seicento.

\begin{tabular}{|c|c|c|c|}
\hline RISM B/I & Titolo della raccolta & Compositori & $\begin{array}{l}\text { RISM B/I della prima } \\
\text { stampa o delle ristampe } \\
\text { successive }\end{array}$ \\
\hline $1600^{5 a}$ & $\begin{array}{l}\text { Flores musicae, hoc est, suavissimae et lepidissimae } \\
\text { cantiones, madrigalia vulgus nominat, una cum } \\
\text { variis pavanis, paduanis, galliardis [...] } \\
\text { (Heidelberg, Voegelin) }\end{array}$ & Lelio Bertani & \\
\hline $1600^{7}$ & $\begin{array}{l}\text { Madrigali pastorali descritti da diversi et posti in } \\
\text { musica da altri tanti autori a sei voci intitolati Il Bon } \\
\text { Bacio di novo ristampati et corretti. } \\
\text { (Venezia, Angelo Gardano) }\end{array}$ & $\begin{array}{l}\text { Lelio Bertani } \\
\text { Costanzo Porta }\end{array}$ & $\begin{array}{l}1594^{6} \\
1604^{10}\end{array}$ \\
\hline $1600^{8}$ & $\begin{array}{l}\text { De floridi virtuosi d'Italia madrigali a cinque voci } \\
\text { ridotti in un corpo. Nuovamente con ogni diligentia } \\
\text { stampati er seguendo l'ordine de suoi toni posti in } \\
\text { luce. } \\
\text { (Antwerpen, Pietro Phalèse) }\end{array}$ & $\begin{array}{l}\text { Giulio Belli } \\
\text { Lelio Bertani }\end{array}$ & \\
\hline $1600^{9}$ & $\begin{array}{l}\text { Spoglia amorosa. Madrigali a cinque voci de diversi } \\
\text { ecc }{ }^{\text {mi }} \text { musici di novo ristampata et con diligentia } \\
\text { corretta. } \\
\text { (Venezia, Angelo Gardano) }\end{array}$ & Costanzo Porta (2) & $1592^{15}$ \\
\hline $1601^{1}$ & $\begin{array}{l}\text { Falsi bordoni omnium tonorum a diversis } \\
\text { eccellentissimis auctoribus modulati, partim plena, } \\
\text { et partim pari voce, cum quatuor, quinque, et sex } \\
\text { vocibus concinendi: ac etiam duobus choris si placet. } \\
\text { [...] } \\
\text { (Venezia, Angelo Gardano) }\end{array}$ & Costanzo Porta (2) & \\
\hline $1601^{5}$ & $\begin{array}{l}\text { Ghirlanda di madrigali a sei voci, di diversi } \\
\text { eccellentissimi autori de nostri tempi. Raccolta di } \\
\text { giardini di fiori odoriferi musicali. Nuovamente posta } \\
\text { in luce. } \\
\text { (Antwerpen, Pietro Phalèse) }\end{array}$ & Lelio Bertani & \\
\hline
\end{tabular}




\begin{tabular}{|c|c|c|c|}
\hline RISM B/I & Titolo della raccolta & Compositori & $\begin{array}{l}\text { RISM B/I della prima } \\
\text { stampa o delle ristampe } \\
\text { successive }\end{array}$ \\
\hline $1601^{6}$ & $\begin{array}{l}\text { Il trionfo di dori descritto da diversi et posti in } \\
\text { musica, da altretanti autori. A sei voci. } \\
\text { (Antwerpen, Pietro Phalèse) }\end{array}$ & Lelio Bertani & $\begin{array}{l}1592^{11} \\
1595^{2} \\
1596^{9} \\
1599^{10} \\
1614^{11} \\
1628^{12}\end{array}$ \\
\hline $1601^{7}$ & $\begin{array}{l}\text { I diporti della villa in ogni stagione spiegati in quattro } \\
\text { canzoni dell' ill. Francesco Bozza Cavaliere, et posti } \\
\text { in musica da diversi famosi autori, a cinque voci, } \\
\text { novamente dati in luce. } \\
\text { (Venezia, Angelo Gardano) }\end{array}$ & Lelio Bertani & \\
\hline $1604^{8}$ & $\begin{array}{l}\text { Musica di diversi eccellentissimi autori. A cinque } \\
\text { voci. Sopra i pietosi affetti, del M. R. P. D. Angelo } \\
\text { Grillo raccolta per il padre D. Massimiano Gabbiani } \\
\text { da Brescia, monaco cassinese. Novamente posta in } \\
\text { luce. } \\
\text { (Venezia, Angelo Gardano) }\end{array}$ & $\begin{array}{l}\text { Giulio Belli } \\
\text { Lelio Bertani (2) } \\
\text { Amadio Freddi } \\
\text { Costanzo Porta }\end{array}$ & \\
\hline $1604^{13}$ & $\begin{array}{l}\text { Fiori musicali a tre voci de diversi eccelentissimi } \\
\text { auttori. Di novo stampati \& seguendo l'ordine de suoi } \\
\text { toni posti in luce. } \\
\text { (Antwerpen, Pietro Phalèse) }\end{array}$ & Giulio Belli (2) & $1618^{10}$ \\
\hline $1607^{4}$ & $\begin{array}{l}\text { La Terza con il Te Deum et Litanie della B. Vergine et } \\
\text { Santi a otto voci di Pietro Lappi fiorentino maestro } \\
\text { della musica di S. Maria delle Gratie in Brescia. } \\
\text { (Venezia, Alessandro Raveri) }\end{array}$ & Lelio Bertani & \\
\hline $1607^{6}$ & $\begin{array}{l}\text { Musarum Sioniar: motectae et psalmi latini, } \\
\text { Michaëlis Praetorij C. apud sereniss. Principem } \\
\text { Henricum Julium ducem Brunsv. \& Lunaeb., chori } \\
\text { musici magistri, IV. V. VI. VII. VIII. IX. X. XII. XVI. } \\
\text { Vocum, choro et organis accommodatae. I. Pars. } \\
\text { (Nürnberg, Abraham Wagenmann) }\end{array}$ & Costanzo Porta & \\
\hline $1607^{8}$ & $\begin{array}{l}\text { Concerti per sonare et cantare. Di Giulio Radino } \\
\text { padovano cioè canzone, \& ricercari a quattro, \& otto, } \\
\text { mottetti, messe, salmi, \& Magnificat, a cinque, sei, } \\
\text { sette, dieci, dodeci, \& sedeci voci. Novamente dati alle } \\
\text { stampe da Gio. Maria Radino suo padre. } \\
\text { (Venezia, Angelo Gardano). }\end{array}$ & $\begin{array}{l}\text { Bartolomeo Favaretto } \\
\text { Amadio Freddi (2) }\end{array}$ & \\
\hline $1607^{9}$ & $\begin{array}{l}\text { Concerti ecclesiastici a una, a due, a tre et a quarto } \\
\text { voci, con il basso continuo per sonar nell'organo, di } \\
\text { Lodovico Viadana, raccolti da fra Danielle de Perugia } \\
\text { minore osservante. Libro secondo, nuovamenti } \\
\text { composti et dati in luce. Opera XVII. } \\
\text { (Venezia, Giacomo Vincenti) }\end{array}$ & Costanzo Porta (2) & \\
\hline
\end{tabular}




\begin{tabular}{|c|c|c|c|}
\hline RISM B/I & Titolo della raccolta & Compositori & $\begin{array}{l}\text { RISM B/I della prima } \\
\text { stampa o delle ristampe } \\
\text { successive }\end{array}$ \\
\hline $1608^{17}$ & $\begin{array}{l}\text { Amilla libro secondo di canzonette a tre voci di Nicolo } \\
\text { Legname padovano sonatore di lauto. Novamente } \\
\text { composte, et date in luce. } \\
\text { (Venezia, Alessandro Raverii) }\end{array}$ & $\begin{array}{l}\text { Bartolomeo Barbarino } \\
\text { Amadio Freddi }\end{array}$ & \\
\hline $1609^{1}$ & $\begin{array}{l}\text { Florilegium sacrarum cantionum quinque vocum pro } \\
\text { diebus dominicis \& festis totius anni, e celeberrimis } \\
\text { nostri temporis musicis,... } \\
\text { (Antwerpen, Pietro Phalèse) }\end{array}$ & Giulio Belli (2) & \\
\hline $1609^{7}$ & $\begin{array}{l}\text { Concerti ecclesiastici a una, a due, a tre et a quarto } \\
\text { voci, con il basso continuo per sonar nell'organo, di } \\
\text { Lodovico Viadana, libro secondo, di nuovo in questa } \\
\text { terza impressione corretto, et ristampato. Opera } \\
\text { XVII. } \\
\text { (Venezia, Giacomo Vincenti) }\end{array}$ & Costanzo Porta & \\
\hline $1609^{15}$ & $\begin{array}{l}\text { Hortus musicalis, variis antea diversorum authorum } \\
\text { Italiae floribus consitus, jam vero latinos fructus, } \\
\text { mira suavitate \& artificio. V. VI. VII. VIII. \& } \\
\text { pluribus vocibus concinendos, pie \& religiose } \\
\text { parturiens. [...] } \\
\text { (Monaco, A. Berg) }\end{array}$ & $\begin{array}{l}\text { Lelio Bertani (2) } \\
\text { Amaio Freddi } \\
\text { Costanzo Porta }\end{array}$ & \\
\hline $1609^{17}$ & $\begin{array}{l}\text { Sonetti novi di Fabio Petrozzi romano, sopra le ville } \\
\text { di Frascati, et altri, posti in musica a cinque voci da } \\
\text { diversi eccellenti musici, con una a otto in fine. } \\
\text { (Roma, Giovanni Battista Robletti) }\end{array}$ & $\begin{array}{l}\text { Lelio Bertani } \\
\text { Amadio Freddi }\end{array}$ & \\
\hline $1610^{10}$ & $\begin{array}{l}\text { Nova Metamorfosi de diversi autori opera del } \\
\text { R.P.F. Geronimo Cavaglieri dell'Ordine di S. Basilio } \\
\text { dell'Armeni. Libro terzo a sei voci. } \\
\text { (Milano, Melchiorre erede di Ag. Tradate) }\end{array}$ & Giulio Belli & \\
\hline $1611^{12}$ & $\begin{array}{l}\text { Symphonia angelica de diversi eccellentissimi authori } \\
\text { a quatro, cinque et sei voci raccolta da Huberto } \\
\text { Waelrant. Nella quale si contengono i più eccellenti } \\
\text { madrigali che hoggide si cantino. Novamente } \\
\text { ritampata. } \\
\text { (Antwerpen, Pietro Phalèse) }\end{array}$ & Lelio Bertani & $\begin{array}{l}1590^{17} \\
1594^{8} \\
1629^{8}\end{array}$ \\
\hline $1612^{5}$ & $\begin{array}{l}\text { Concerti ecclesiastici a una, a due, a tre, \& a quatro } \\
\text { voci, con il basso continuo per sonar nell'organo di } \\
\text { Lodovico Viadana libro secondo di nuovo in questa } \\
\text { quarta impressione corretto \& ristampato. Opera } \\
\text { XVII. } \\
\text { (Venezia, GiacomoVincenti) }\end{array}$ & Costanzo Porta & \\
\hline $1613^{2}$ & $\begin{array}{l}\text { Promptuarii musici, sacras harmonias sive motetas } \\
\text { V. VI. VII. \& VIII. Vocum, e diversis, iisque } \\
\text { clarissimis hujus et superioris aetatis authoribus } \\
\text { collectas comprehendentis, pars tertia: [...] } \\
\text { (Strasbourg, Karl Kieffer) }\end{array}$ & $\begin{array}{l}\text { Giulio Belli (2) } \\
\text { Costanzo Porta (2) }\end{array}$ & \\
\hline
\end{tabular}




\begin{tabular}{|c|c|c|c|}
\hline RISM B/I & Titolo della raccolta & Compositori & $\begin{array}{l}\text { RISM B/I della prima } \\
\text { stampa o delle ristampe } \\
\text { successive }\end{array}$ \\
\hline $1613^{3}$ & $\begin{array}{l}\text { Canoro pianto di Maria Vergine sopra la faccia } \\
\text { di Christo estinto in poesia del Rev. abbate } \\
\text { Grillo raccolta per D. Angelico Patto accademico } \\
\text { giustiniano, et posta in musica da diversi auttori con } \\
\text { un dialogo, et madrigale tramutati da l'istesso. A una } \\
\text { voce da cantar nel chitarone o altri instromenti simili. } \\
\text { Novamente stampati. } \\
\text { (Venezia, Bartolomeo Magni) }\end{array}$ & $\begin{array}{l}\text { Bartolomeo Barbarino (6) } \\
\text { Amadio Freddi (2) }\end{array}$ & \\
\hline $1613^{6}$ & $\begin{array}{l}\text { Opera omnia sacrorum concertum I. II. III. et } \\
\text { IV. vocum, iam convenineter collecta. Cum basso } \\
\text { continuo et generali organo adplicato; [...] } \\
\text { (Frankfurt, N. Stein) }\end{array}$ & Costanzo Porta & \\
\hline $1613^{10}$ & $\begin{array}{l}\text { Il Parnasso, madrigali de diversi eccellentissimi } \\
\text { musici a sei voci nuovamente raccolti \& dati in luce. } \\
\text { (Antwerpen, P. Phalèse) }\end{array}$ & Amadio Freddi (2) & \\
\hline $1613^{13}$ & $\begin{array}{l}\text { Rest musicalisches Streitkränzleins: hiebenvorn von } \\
\text { den allerfürtrefflichsten }[. . .] \\
\text { (Nürnberg, B. Scherff) }\end{array}$ & $\begin{array}{l}\text { Luigi Balbi } \\
\text { Lelio Bertani } \\
\text { Costanzo Porta }\end{array}$ & \\
\hline $1615^{3}$ & $\begin{array}{l}\text { Musia vaga et artificiosa continente motetti con } \\
\text { oblighi, et canoni diveri, tanto per quelli, che si } \\
\text { dilettano sentire varie curiosità, quanto per quelli, } \\
\text { che vorranno professare d'intendere diversi studii } \\
\text { della musica. Di D. Romano Micheli romani. } \\
\text { Novamente composta, et data in luce. } \\
\text { (Venezia, Giacomo Vincenti) }\end{array}$ & Luigi Balbi & \\
\hline $1615^{13}$ & $\begin{array}{l}\text { Parnassus musicus Ferdinandaeus in quo musici } \\
\text { nobilissimi, qua suavitate, qua arte prorsus } \\
\text { admirabili \& divina ludunt 1. 2. 3. 4. 5. vocum, a } \\
\text { Joanne Baptista Bonometti bergomate serenissimi } \\
\text { Ferdinandi archiducis Austriae \&c. musico congestus } \\
\text { (Venezia, Giacomo Vincenti). }\end{array}$ & $\begin{array}{l}\text { Bartolomeo Barbarino } \\
\text { Giovanni Ghizzolo (2) }\end{array}$ & \\
\hline
\end{tabular}

\section{Bibliografia}

\section{EDIZIONI ANTICHE}

Gualtieri, Antonio. Il secondo libro de mottetti a una e due voci con li salmi a tre voci con basso per l'organo. Venezia, erede di Angelo Gardano, 1612.

Viadana, Ludovico. Concerti ecclesiastici a una, a due, a tre et a quatro voci, con il basso continuo per sonar nell'organo. Venezia: Giacomo Vincenti, 1609. 


\section{LETTERATURA}

Antonicek, Theophil, ed. Parnassus musicus Ferdinandaeus herausgegeben von Giovanni Battista Bonometti (1615). Denkmäler der Tonkunst in Österreich, 159. Graz: Akademische Druck- u. Verlagsanstalt, 2015.

Cattin, Giulio e Maria Nevilla Massaro. "La musica e le istitutzioni musicali nelle città di terraferma". In Storia della Cultura Veneta, 449-454. Il Seicento, 4/1. Vicenza: Neri Pozza Editore, 1983.

Colussi, Franco. “Gualtieri, Antonio”. In Nuovo Liruti: Dizionario biografico dei friulani 2, 1385-1388, Udine: Forum, 2009.

___ . "Tracce di musica policorale in alcuni centri del Friuli storico tra Cinque e Seicento". In La musica policorale in Italia e nell'Europa centro-orientale fra Cinque e Seicento, a cura di Aleksandra Patalas e Marina Toffetti, 101-158. Venezia: Fondazione Ugo e Olga Levi, 2012.

Comparin, Chiara. "Antonio Gualtieri (Monselice, 1574-1661). Opere sacre e profane." Diss. dottorale, Università degli Studi di Padova, 2015.

Dizionario Enciclopedico della Musica e dei Musicisti, diretto da Alberto Basso, Le biografie, vol. 3. Torino: UTET, 1985-1990.

Eitner, Robert. Bibliographisch-bibliographisches Quellen-Lexicon der Musiker und Musikgelehrten christlicher Zeitrechnung bis Mitte des neunzehnten Jahrhunderts. Vol. 3-4. Graz: Akademische Druck- u. Verlagsanstalt, 1960.

Garbelotto, Antonio. Un vescovo musicista nel 500: Marco Cornaro. Padova: Società Cooperativa Tipografica, 1954.

Lovato, Antonio. "La musica sacra nell'attività pastorale del vescovo di Padova Marco Corner (1557-1625)”. Studia patavina 34 (1987): 29-50.

. "Musica e liturgia nella collegiata di S. Giustina". In Monselice nei secoli, a cura di Antonio Rigon, 231-249. Treviso: Canova, 2009.

Morche, Gunther. "Gualtieri, Antonio". In Die Musik in Geschichte und Gegenwart, seconda edizione a cura di Ludwig Finscher, Personenteil, 8:140-142. Kassel: Bärenreiter; Stuttgart: Metzler, 1998.

Morelli, Arnaldo. "Il Seicento". In Storia della Musica al Santo di Padova, 93-106. Fonti e Studi per la Storia del Santo a Padova, 10. Vicenza: Neri Pozza Editore, 1990.

Müller, Josef. Die musikalischen Schätze der Staats- und Universitätsbibliothek zu Königsberg i. Pr. Bonn: Adolph Marcus, 1870.

Passadore, Francesco. Musica e musicisti a Rovigo tra Rinascimento e Barocco. Rovigo: Minelliana, 1987.

Roche, Jerome e Elizabeth Roche. "Gualtieri, Antonio". In The New Grove Dictionary of Music and Musicians, seconda edizione a cura di Stanley Sadie, 10:472-473, Londra: Macmillan, 2001.

Scattolin, Pier Paolo. "La cappella musicale del duomo di Montagnana (1592-1682). Profilo storico”. Rassegna Veneta di Studi Musicali, 15-16 (1999-2000): 287-374. 


\section{IL SECONDO LIBRO DE MOTTETTI A UNA E DUE VOCI ANTONIJA GUALTIERIJA IN PARNASSUS MUSICUS FERDINANDAEUS}

\section{Povzetek}

Leta 1612, samo tri leta pred izidom antologije Parnassus musicus Ferdinandaeus, je v Benetkah zagledala luč sveta druga knjiga motetov za enega do dva glasova in basso continuo Antonija Gualtierija. Gualtieri je delal kot musices magister in kapelnik v Furlaniji in na Beneškem, med kraji Monselice, Montagnana in Benetke. Nekoliko pozneje je Gualtieri izdal še eno knjigo motetov za nekaj glasov in basso continuo: Motetti a una, doi, tre et quarto voci con le littanie della Beata Vergine a Quattro (Benetke, 1630).

Gualtierijeva ustvarjalnost v celoti odseva kulturno klimo zgodnjega 17. stoletja. Začel je s polifonimi moteti, v katerih je jasno zaznaven vpliv najpomembnejših padovanskih mojstrov. Potem je v svojih duhovnih delih slogovno napredoval do bolj izdelanih motetov za nekaj glasov in basso continuo; nekateri od teh so bili obogateni z glasovi za glasbila, kot so violine in pozavne. $V$ šestintridesetih motetih za enega do štiri glasove in continuo, objavljenih v zbirkah iz let 1612 in 1630, je Gualtieri dosegel precej moderen koncertantni slog in je opustil polifono oblikovanje iz svojega prvotiska Motecta octonis vocibus (Benetke, 1604).

$\mathrm{V}$ razpravi so osvetljene glavne značilnosti Gualtierijeve zbirke iz leta $1612 \mathrm{v}$ primerjavi z njegovimi moteti, objavljenimi tri leta pozneje v antologiji Parnassus musicus Ferdinandaeus. Ti moteti kažejo podobno zgradbo in uporabo kompozicijskih sredstev. V Gualtierijevih motetih tako najdemo splošne značilnosti in principe, kot so jih uporabljali tudi drugi sodobni skladatelji. Uporabljal je inovativne tehnike, ki se jih je naučil $\mathrm{v}$ Padovi in v Benetkah, ter jih prilagodil praktičnim izvajalskim potrebam manjših glasbenih središč, kjer je deloval.

Primerjava Gualtierijevih motetov z mnogimi moteti zbirke Parnassus razkrije podobnosti s slogom skladateljev, ki so delovali po drugih pokrajinah italijanskega polotoka in so njihova dela prodrla do Gradca. 